\title{
Lidil
}

Revue de linguistique et de didactique des langues

56 | 2017

Grammaires et littéracies

\section{Quelles pratiques littéraciées sont mobilisées par les futurs professeurs des écoles lorsqu'ils « font de la grammaire »?}

What Literate Practices Are Mobilized by Future School Teachers When They

"Do Grammar"?

\section{Belinda Lavieu-Gwozdz et Thierry Pagnier}

URL : http://journals.openedition.org/lidil/4806

DOI : 10.4000/lidil.4806

ISSN : $1960-6052$

Éditeur

UGA Éditions/Université Grenoble Alpes

Édition imprimée

ISBN : 978-2-37747-024-2

ISSN : $1146-6480$

\section{Référence électronique}

Belinda Lavieu-Gwozdz et Thierry Pagnier, « Quelles pratiques littéraciées sont mobilisées par les futurs professeurs des écoles lorsqu'ils « font de la grammaire »? », Lidil [En ligne], 56 | 2017, mis en ligne le 01 novembre 2017, consulté le 19 avril 2019. URL : http://journals.openedition.org/lidil/4806 DOI : 10.4000/lidil.4806

Ce document a été généré automatiquement le 19 avril 2019

(C) Lidil 


\title{
Quelles pratiques littéraciées sont mobilisées par les futurs professeurs des écoles lorsqu'ils « font de la grammaire »?
}

\author{
What Literate Practices Are Mobilized by Future School Teachers When They \\ "Do Grammar"?
}

Belinda Lavieu-Gwozdz et Thierry Pagnier

\section{Introduction}

1 La masterisation et plus largement l'ensemble des réformes successives de la formation des futurs professeurs des écoles (PE) ont remis sur le devant de la scène la question du rapport au savoir qu'impliquent les processus d'apprentissage / enseignement.

2 Un rapide sondage interrogeant nos étudiants de master 1 MEEF (Métiers de l'enseignement, de l'éducation et de la formation) sur la manière dont ils perçoivent la grammaire montre qu'ils entretiennent une aversion pour celle-ci et que beaucoup appréhendent tant son apprentissage que son enseignement.

\section{Malentendus sociocognitifs et littéracies}

Le travail présenté ici s'inscrit dans une recherche menée depuis deux ans au sein d'un laboratoire qui centre ses préoccupations sur la question des inégalités d'apprentissage à l'école, et notamment sur le rôle du langage et des discours dans la construction de ces inégalités. Au cœur de ces travaux figure le concept de co-construction des inégalités scolaires (Bautier \& Rayou, 2009 ; Rochex \& Crinon, 2011; Delarue-Breton, 2012, 2014 et 2015). Les inégalités entre élèves, et donc les difficultés importantes, conduisant parfois à l'échec de certains d'entre eux, s'analysent comme étant le produit d'une rencontre entre 
des modes de socialisation familiale peu connivents des pratiques littéraciées scolaires, et des pratiques pédagogiques qui tiennent trop - ou trop peu - compte de ces écarts entre élèves au cours des activités d'apprentissage en classe. Nous proposons ici de transposer cette notion de co-construction et de malentendus au contexte de formation universitaire des futurs professeurs des écoles, inscrits en première année de master MEEF. Le concept de malentendu sociocognitif invite à prendre en compte le travail interprétatif de l'apprenant, qui risque de masquer les significations à construire. C'est cette préoccupation qui nous a conduits à nous intéresser aux literacy studies et à la manière dont les concepts proposés nous permettent de mettre en évidence le sens que les étudiants ont effectivement construit des situations d'enseignement-apprentissage de la grammaire qu'ils ont vécues, qu'ils vivent en tant qu'apprenant et qu'ils vivront en tant qu'enseignant.

4 Pour penser ces malentendus sociocognitifs, nous proposons d'inscrire cette réflexion dans le champ des recherches sur les littéracies.

5 La maitrise de l'écriture (comprise globalement) intègre les savoirs et savoir-faire, mais aussi la culture du lire-écrire, c'est-à-dire les fonctions et les usages de l'écrit qui cristallisent en quelque sorte les rapports entre langage et cognition et langage et pouvoir. L'écriture ne se réduit donc pas, dans l'approche que nous proposons, à sa dimension matérielle.

6 À la suite de Fraenkel et Mbodj (2010), on peut considérer que l'avantage du terme de « littéracie » est justement de référer indissociablement à l'écriture comme technologie et comme culture impliquant des savoirs, des savoir-faire et des postures. Nous partageons l'idée que le concept de littéracie désigne « des configurations cohérentes de pratique de l'écrit» (Barton \& Hamilton, 2010, p. 48). Cet ancrage dans la lignée des propositions de Hymes $(1962,1972,1984)$ vise à proposer un cadre d'analyse qui s'intéresse aux manières différenciées dont les étudiants se représentent et donnent sens à ce qu'ils identifient comme des situations d'enseignement-apprentissage de la grammaire. Il s'agit avant tout de chercher à adopter le point de vue des locuteurs, en l'occurrence des étudiants, futurs PE. Notre objectif est de donner à voir non pas les lacunes des étudiants qu'il s'agirait de combler, mais la cohérence d'une configuration des usages et des fonctions de l'écrit jugée adéquate (c'est-à-dire attendue) par les étudiants dans ces situations. Dans ce cadre, les difficultés des étudiants sont pensées comme les conséquences d'une série de malentendus sur ce qu'il s'agit de faire, comment le faire et avec quoi le faire. Nous défendons effectivement l'idée que les pratiques des étudiants relèvent de la littéracie lorsqu'ils «font de la grammaire» mais que les compétences littéraciées qu'ils mobilisent relèvent d'une littéracie ordinaire et non de la littéracie scolaire.

7 Dans cette perspective, notre utilisation du concept de literacy event (Barton \& Hamilton, 2010, p. 47) inscrit notre recherche dans la lignée des réflexions méthodologiques, (notamment Hymes, 1962) visant à identifier les situations de communication telles que les locuteurs les distinguent. Appliqué à notre terrain et aux locuteurs observés, cela nous a d'abord conduits à nous intéresser à ce que les étudiants identifient comme des moments d'enseignement-apprentissage de la grammaire. Il s'agit donc de ne pas s'en tenir au discours classificateur institutionnel, mais bien d'interroger la manière dont leur expérience (langagière et cognitive) les conduits à distinguer ces «évènements de littéracie ». 
8 Nous parlerons ici de pratiques littéraciées dans la mesure où il s'agit non seulement d'acquérir des formes écrites, mais aussi un rapport à la langue, à son écriture, au résultat des transformations sociales et cognitives que l'usage de l'écrit a produit dans la société et qui ont conduit à étendre le rapport symbolique et médiatisé à la langue jusqu'aux objets du monde et à l'expérience elle-même, y compris dans les situations d'échange oral. Certaines manières de se saisir de l'écrit, certaines compétences d'écriture ne sont pas toujours celles attendues en contexte scolaire, bien que relevant de la littéracie. Il s'agit donc de faire une place à une littéracie ordinaire, celle que l'on observe notamment en prenant en compte les échanges scripturaux contemporains (on échange davantage d'écrit, on fait plus de choses différentes avec l'écrit).

9 À la suite de Hymes, nous considérons les litteracy event comme des cas (bien) particuliers de speech event. En l'occurrence, les literacy event que les étudiants identifient au sein des activités et des temps scolaires comme relevant de la grammaire font apparaitre des écarts bloquant la mise en œuvre efficiente d'une réflexion grammaticale.

10 En effet, les situations d'enseignement-apprentissage de la grammaire qu'identifient les étudiants sont bien des situations qui mettent en jeu des compétences littéraciées telles que la compétence métalangagière ou la manipulation de listes et de stocks d'étiquettes voire de procédures d'appariement. Il s'agit, par ailleurs, de situations dans lesquelles l'écrit structure bien leurs pratiques. C'est bien avec, par et sur de l'écrit qu'ils réalisent l'activité.

11 Au-delà des compétences du lire-écrire, c'est en effet un rapport réflexif sur la langue qui est engagé. En l'occurrence tous les étudiants sont en mesure de placer la langue en position d'objet de discours. Toutefois, l'activité grammaticale (visée) implique de faire de la langue un objet, mais un objet d'un type particulier : un objet d'analyse, c'est-à-dire un objet sur lequel va se développer un discours de nature scientifique, qui vise la construction de signification. En l'occurrence, lors de ces séances de "grammaire », il s'agit de développer un discours « scientifique » sur la langue au sens de Bautier (2002) et Delarue-Breton (2012a). En somme, la procédure est celle de l'analyse scientifique avec des procédures de vérification et des catégories d'analyse.

Or, les étudiants ne semblent pas inscrire ces «évènements de littéracie » dans des pratiques spécifiques de littéracie que constitue justement ce que l'on nomme la littéracie scolaire. Nous désignons par littéracie scolaire non seulement les usages de l'écrit, les supports de travail, mais aussi les exigences de lecture, de raisonnement et de connaissances qui supposent une familiarité avec la fréquentation de ces écrits scolaires.

13 Méthodologiquement, il s'est agi de partir des literacy events pour définir les literacy practices dans lesquelles elles prennent sens. En l'occurrence, il s'agit de caractériser les pratiques littéraciées effectives avec les pratiques littéraciées attendues lors des situations d'enseignement-apprentissage de la grammaire.

Notre travail vise donc à montrer que la source du malentendu tient, d'une part, à la manière dont les étudiants inscrivent ou non ces évènements de littéracie dans le champ de la littéracie scolaire et, d'autre part, au caractère plus ou moins invisible de ce malentendu dans les situations scolaires pour les étudiants comme les formateurs. 


\section{Question de recherche, méthodologie et corpus}

\subsection{Question de recherche}

15 À l'articulation avec le travail d'Hubert (2016) dans lequel la dimension psychologique du rapport normatif à la grammaire est efficacement mise en évidence et avec celui d'Ulma (2016) qui considère la « conceptualisation » des « notions » comme un moyen de dépasser les difficultés rencontrées par les étudiants, notre recherche interroge les conditions d'une construction socialement différenciée d'un rapport « second " au savoir engageant inextricablement les dimensions cognitives, psychiques et langagières de l'individu.

Une des hypothèses que nous avançons pour expliquer ce désamour et cette insécurité linguistique et professionnelle vis-à-vis de la grammaire tient donc à la perception fragmentaire et erronée des enjeux d'apprentissage des activités grammaticales que les étudiants, futurs PE, ont construite au fil de leur scolarité primaire, secondaire et universitaire.

17 Nous cherchons à voir comment les habitudes scolaires et cognitives construites lors des " cours de grammaire " dans le cadre de l'école élémentaire et au secondaire ont une incidence sur le rapport à la langue et à la grammaire qu'entretiennent les étudiants. Nous faisons l'hypothèse que ce sont ces habitudes cognitives et scolaires durablement installées, qui gênent voire empêchent les apprentissages grammaticaux dans le long terme.

\subsection{Méthodologie}

18 Cette recherche s'appuie sur un protocole de recueil de données réalisé auprès d'une cohorte de 500 étudiants inscrits en master MEEF et organisé en deux temps : un test en ligne et des entretiens en binôme voire trinôme avec un enseignant-chercheur ou un formateur de l'ESPE. Un double traitement de nos données a été mené : qualitatif pour les catégories d'analyse et quantitatif pour l'analyse globale des performances des étudiants.

19 Nous accédons aux performances des étudiants par l'analyse quantitative des réponses aux questions du test en ligne. Le traitement statistique a permis de faire apparaitre des fréquences et des corrélations significatives dans les choix opérés par les étudiants. Par ailleurs, une analyse des réponses aux questions ouvertes (Comment le savez-vous ?) du test et des entretiens avec les étudiants a permis de développer une approche plus qualitative en interrogeant notamment les processus de nomination (Pagnier, 2010) observables dans le discours des étudiants.

Nos corpus ${ }^{1}$ sont constitués à partir des données suivantes :

- le test de grammaire initial en ligne qui se fait sur site durant 1 heure, l'ensemble des réponses des étudiants ayant été traité et recueilli informatiquement ;

- l'enregistrement audio des étudiants lors de l'entretien mené par les formateurs à partir du guide d'entretien ;

- une fiche individuelle détaillée reprenant l'enjeu de chacune des questions du test de grammaire initial et les hypothèses d'analyse du choix que l'étudiant a réalisé (et qui sert de support à ce travail d'entretien). 
21

recherche nous a conduits à questionner conjointement l'utilisation du tes diagnostique et l'articulation entre les réponses aux questions et les procédures auxquelles les étudiants recourent pour y répondre. Nous avons choisi de nous intéresser moins à la maitrise des exercices «scolaires » traditionnels (essentiellement de repérage de natures et de fonctions) qu'aux représentations de la grammaire et de la langue des étudiants et plus précisément à la manière dont les étudiants ont plus ou moins achevé la construction de notions grammaticales comme concept et outil d'analyse de la langue.

\subsection{Le corpus}

\subsubsection{Des tests en ligne}

Le choix des questions lors de la première phase du protocole, à savoir la phase de passation du test, s'organise en trois volets :

- un premier volet interroge sur des questions d'identification (exercices scolaires, type concours) et porte sur le repérage de nature, fonction des mots ainsi que sur l'accord des participes passés ;

- un second volet porte sur les questions de verbalisation des procédures et des savoirs grammaticaux (inspiré des travaux de Elalouf, 2005) et se consacre à la catégorie nom/verbe lorsqu'ils sont homophones (marche), à l'opposition complément de verbe/complément circonstanciel (manger la nuit vs manger une pizza), aux indices linguistiques dans la compréhension en lecture, au rapport syntaxe/sémantique (segmentation), ainsi qu'à la notion du couple phrase simple/phrase complexe.

- un troisième volet interroge les étudiants (sur le mode déclaratif) sur les représentations de la langue, sur son analyse et son enseignement.

\subsubsection{Des entretiens}

À l'issue de ce test initial automatisé réalisé, les étudiants reçoivent une fiche individuelle récapitulant les réponses aux questions. C'est à partir de ce document et du guide d'entretien que se déroulent les entretiens (en binôme ou trinôme d'étudiants) qui interrogent les étudiants sur leurs manières de faire lorsqu'ils doivent répondre à des tests en ligne ou à des questions de concours lors de la première phase du protocole (retour sur des questions précises du test, sur la finalité de celui-ci et sur les procédures²). Ces entretiens visent avant tout à questionner les procédures et les conceptions des étudiants. Ils sont l'occasion de mettre en évidence les enjeux d'apprentissage et de signaler aux étudiants comme aux formateurs si les enjeux ont été ou non perçus.

La réalisation des tests a donc placé les étudiants devant cette tâche - peu réussie lors de cette réalisation individuelle - de verbalisation des procédures et des savoirs grammaticaux. En revanche, cette préparation dans le cadre d'un test a sans aucun doute participé à la construction effective d'une analyse collective lors des entretiens en binôme ou trinôme. Nous reviendrons en conclusion sur la manière dont ceux-ci ont constitué des moments clés dans l'explicitation des procédures, des catégories et donc de l'activité grammaticale elle-même en offrant un espace interactionnel préparé permettant effectivement l'appropriation individuelle d'une analyse collectivement construite. 


\section{Analyses et constat}

Au-delà du sens que les étudiants donnent aux catégories grammaticales (sujet, phrase verbe...), nous cherchons aussi à identifier le sens qu'ils donnent à des opérations linguistiques (segmentation, suppression, déplacement, commutation) et aux enjeux d'apprentissage de l'activité grammaticale en analysant comment ils utilisent ces opérations et ces catégories. L'ensemble des données recueillies et analysées indique que les étudiants de master MEEF ont acquis des compétences littéraciées, mais ne mobilisent pas celles qui sont attendues d'un futur professeur des écoles.

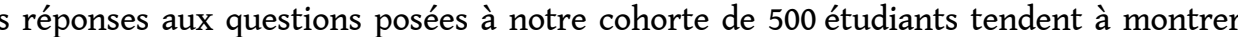
qu'ils ont construit des connaissances grammaticales « fragiles » à propos des notions de groupe du nom, groupe du verbe, de phrases subordonnées et de phrases simples. Pour préciser ce que d'aucuns qualifieraient de "fragiles", nous essaierons de contraster les manières dont les catégories sont construites et structurées par les étudiants avec celles que proposent les sciences du langage. Le débat sur la vulgarisation ou plutôt la transposition didactique des savoirs grammaticaux et la manière dont les élèves s'en emparent effectivement n'est pas nouveau. Il ne s'agit donc pas de dénoncer une transposition didactique plutôt qu'une autre. Notre réflexion vise à identifier précisément la manière dont les étudiants donnent sens aux catégories qu'ils mobilisent.

Les écarts ne concernent pas uniquement la maitrise d'un métalangage scientifique, mais impliquent, expliquent et s'expliquent par la représentation des étudiants concernant l'activité grammaticale tant dans ses finalités que dans les catégories et les procédures cognitives, langagières et métalangagières qu'elle implique. Ainsi les trois paragraphes qui suivent illustrent le fait que les étudiants ne perçoivent pas la finalité des exercices de grammaire, ce qui engendre le recours à la fois à des procédures inadaptées et à la manière ordinaire de catégoriser qui ne relève pas de la catégorisation scientifique (ou du moins scolaires) que l'on pourrait attendre d'étudiants de master, futurs professeurs des écoles.

\subsection{Une finalité strictement autotélique des exercices de grammaire}

La perception que les étudiants ont des activités universitaires et notamment des exercices de grammaire n'échappe pas à la dimension factuelle que les travaux d'Escol ont pu mettre en évidence. En effet, d'après Delarue-Breton (2012b), les activités scolaires se caractérisent par la dualité de leur finalité. Ainsi, à l'école il ne s'agit pas (seulement) de faire (c'est-à-dire d'effectuer une tâche) ce qu'on demande de faire, l'élève doit (en plus) percevoir ce que Delarue-Breton (2012b, p. 121-122) appelle la "visée allotélique » des dispositifs, c'est-à-dire une finalité seconde à la réalisation de la tâche. Pour illustrer cette double finalité, nous pouvons reprendre l'exemple en dehors du cadre scolaire de la partie de tennis (Belin, 2002). En effet, jouer au tennis peut avoir comme finalité de prendre du plaisir, finalité interne dite autotélique qui s'épuise dans la réalisation même de la tâche, mais jouer au tennis peut aussi et en même temps avoir comme finalité d'améliorer son classement international, c'est-à-dire une visée externe appelée « allotélique » (Delarue-Breton, 2012b, p. 121-122).

Au-delà du cadre qui rompait avec les habituels exercices, les premiers résultats montrent que pour une part importante des étudiants l'activité grammaticale demeure 
une activité tendue par une finalité que l'on qualifierait volontiers d'autotélique (Belin, 2002; Delarue-Breton, 2012a) tant elle s'épuise dans la réalisation même de l'activité : faire de la grammaire, c'est étiqueter.

Ainsi, par exemple, de nombreux étudiants ont rencontré des difficultés à répondre aux items interrogeant les relations entre l'analyse de la structure de la phrase et le sens construit par l'énoncé. Lorsque les étudiants ont à sélectionner la segmentation grammaticale qui correspond à l'un des deux sens d'une phrase ambigüe comme Mon voisin a rapporté un vase de Chine, seule la moitié d'entre eux sélectionne la bonne segmentation. La segmentation syntaxique d'une phrase ne sert donc pas, pour les étudiants, à construire le sens d'une phrase. De même, lorsqu'il s'agit de sélectionner « la ou les» fonctions possibles d'un syntagme dans une phrase ambigüe comme Mon voisin rêve à ses vacances au bord de la mer, $25 \%$ des étudiants proposent une segmentation mot à mot et $30 \%$ s'étonnent que cet énoncé puisse recevoir deux segmentations possibles qui véhiculent deux sens différents, possibilité pourtant explicitement formulée dans la consigne $^{3}$. La finalité autotélique d'étiquetage est réalisée dès la première étiquette posée et ne nécessite à leurs yeux aucun prolongement quelle que soit la consigne.

Lorsque nous leur posons explicitement la question À quoi sert la grammaire ?, 98 \% d'entre eux indiquent qu'elle sert (entre autres) à lire et à comprendre un texte. Néanmoins lorsqu'on qu'on leur demande d'identifier le sexe de deux personnages dans une phrase comme Claude avait appelé Dominique plusieurs fois pour avoir de ses nouvelles. C'était son amie tout de même. Il ne pouvait être question entre eux de préséance ou de politique inutile... pour près de la moitié des étudiants (223 sur 500) la réponse à cette question est qu'on ne peut pas savoir le sexe de Claude. Les étudiants ne perçoivent donc (si on ne le leur signale pas explicitement) pas qu'il faut s'appuyer sur des indices linguistiques fins (amie et eux) pour pouvoir répondre à cette question de compréhension en lecture. On constate là encore comme les réponses «formelles » des étudiants peuvent en surface correspondre à la réponse attendue.

32 L'exercice de grammaire proposé dans le cadre de cette recherche a lui-même été interprété de manières très différentes. En effet, la majorité des étudiants ayant eu une note inférieure à la moyenne des autres étudiants ont interprété la finalité de cet exercice comme une évaluation de leurs connaissances, tandis que la majorité de ceux qui ont obtenu plus que la moyenne ont essentiellement pointé le travail de verbalisation des procédures et des savoirs que ce test introduisait.

\subsection{Les procédures mobilisées relèvent d'un discours premier}

33 Lorsqu'on interroge les étudiants sur les procédures mises en œuvre pour répondre aux questions du test par des questions ouvertes du type «Les mots en majuscule sont tous de la même nature sauf un. Lequel ? Comment l'avez-vous trouvé ?" ", parmi les 500 étudiants ayant répondu à cette question qui vise le "comment», $5 \%$ répondent sur l'activité mentale très générale : «Par élimination, en lisant toutes les phrases afin de repérer les points communs ", "j'ai lu chaque phrase ", « en cherchant", « en réfléchissant ", «à l'intuition ». Nous pouvons distinguer dans les déclarations des manières de faire des étudiants, deux catégories : une première catégorie ("au feeling ", à l'intuition) qui ne relève pas d'un processus de réflexion dans la mesure où l'activité cognitive n'est pas signalée et que donc nous ne pouvons pas interroger, faire verbaliser lors de l'entretien, et une seconde catégorie qui signale des processus de réflexion (en cherchant, en 
réfléchissant, en lisant) sur laquelle nous avons questionné les étudiants lors de l'entretien en binôme, par exemple en essayant de savoir ce que représente pour lui l'activité de recherche ${ }^{5}$ ou encore l'activité de lecture ${ }^{6}$ dans le cadre d'une activité grammaticale.

Une troisième catégorie d'étudiants - la majorité (à peu près $80 \%$ ) - répond à cette question sur les procédures en s'appuyant sur les catégories de la langue (nom et verbe): "c'est un verbe et il décrit l'action », «j'ai vu que c'était le seul qui était un verbe ». La verbalisation des procédures est une activité rarement demandée aux étudiants si bien qu'ils sont démunis lorsqu'on les interroge à ce propos. Lors des entretiens, nous sommes revenus sur ces questions ouvertes en comment et les étudiants déclarent qu'ils ne sont pas habitués à ce genre de questions, que cela les a déroutés, qu'ils ne voyaient pas ce qu'on cherchait avec ces questions. Il semble que la prise en compte des procédures, des manières de faire, ne relève pas du cours de français alors qu'en mathématiques, par exemple, ils trouvent normal qu'on les interroge sur la manière dont ils s'y sont pris pour construire une figure géométrique ou obtenir le résultat d'une opération.

De manière générale, les procédures sont principalement marquées par le recours systématique aux questions d'ordre sémantique en qui est-ce qui ?, à qui ?, comment ?, quoi ? , qui est effectivement une procédure enseignée dans l'élémentaire et le secondaire, mais le plus souvent parmi d'autres procédures. Ici elle fonctionne isolément faisant ainsi reposer la catégorisation sur un jugement de typicalité censément suffisant. Les étudiants n'envisagent d'ailleurs que rarement des prolongements.

Les procédures sont ainsi primarisées en ce sens qu'elles ne sont pas ressaisies, retravaillées, secondarisées dans une perspective d'apprentissage de la langue et de son fonctionnement, mais sont extraites et mises en fonctionnement dans un discours premier, comme on pourrait le faire dans un échange ordinaire, conversationnel.

La focalisation sur le résultat de l'analyse grammaticale conduit à des opérations cognitives d'un ordre très différent de celui qui est attendu. Ce qui doit être fait avec les textes suppose d'identifier la finalité allotélique de l'activité7. La nature des textes proposés n'est pas identifiée par les étudiants qui n'utilisent pas la consigne et le corpus comme l'on pourrait s'y attendre. Ainsi, lorsqu'on leur demande de donner la nature d'expansions nominales et qu'on souligne le noyau de ces expansions (à savoir le nom), ceux-ci donnent la nature du mot souligné dans $80 \%$ des cas. Là encore le formatage des exercices de grammaire au niveau sémiotique (soulignement, encadrement, verbe en rouge, etc.) auquel les étudiants ont été entrainés durant leur scolarité entre en conflit avec l'activité grammaticale qu'on leur demande. La grande majorité des étudiants cherchent donc à répondre à l'exercice (à effectuer une tâche) indépendamment de la consigne sur la base de ce qu'ils interprètent comme des indices sémiotiques (le soulignement ici) présents dans le corpus. Plus étonnant encore, lorsqu'au cours de l'entretien on leur demande l'intérêt d'avoir répondu que le mot souligné était un nom alors que la question porte sur l'expansion du nom (et que donc le noyau est nécessairement un nom), près de $40 \%$ ne comprennent pas immédiatement que leur réponse est incongrue. 


\subsection{Les catégories mobilisées non reconfigurées dans un discours second}

de la phrase ("masque ») mettant en jeu un effet d'homophonie entre nom (le masque) et verbe (il masque). Cette focalisation sur le contexte étroit n'explique toutefois pas entièrement la difficulté. En effet, on peut déduire des réponses fournies que cette focalisation se couple, là encore, d'une catégorisation de l'unité « masque » qui relève de la vérification de la présence vs de l'absence dans le stock des noms (ou des verbes) en l'occurrence.

42 Lorsqu'il s'agit de justifier l'identification d'une phrase simple parmi des phrases complexes, les étudiants construisent dans leur discours une catégorie " phrase simple » qui correspond à une sorte de primarisation du discours second que constitue le discours de l'analyse grammaticale. En effet, la dénomination « phrase simple » n'est pas identifiée comme relevant de la nomenclature grammaticale et n'a donc pas le statut de dénomination mais de désignation, au sens de Kleiber ${ }^{8}$, dans le discours des étudiants. Moins de $20 \%$ ont d'ailleurs identifié la phrase simple dans le corpus. Par ailleurs, $10 \%$ de 
ceux qui ont fourni la bonne réponse déclarent ne pas savoir comment ni pourquoi ils ont sélectionné cette réponse. Les $80 \%$ d'étudiants n'ayant pas fourni la bonne réponse justifient leur choix par la ponctuation (comme d'ailleurs $5 \%$ des étudiants ayant fourni la bonne réponse) et plus généralement sur des indices de surface stéréotypiques, mais non nécessaires et non suffisants. Ainsi, faute de renvoyer à un concept défini par une série de propriétés fonctionnant comme des conditions nécessaires et suffisantes d'appartenance à une catégorie, les étudiants mobilisent des caractéristiques que leur expérience les a conduits à identifier comme des propriétés souvent partagées par les membres de la catégorie et peu par les membres des autres catégories. Ce faisant, la dénomination "phrase simple » n'est pas repérée et beaucoup d'étudiants en font une désignation dans laquelle l'adjectif simple a son sens le plus courant dans le français ordinaire. Une phrase simple est donc "une phrase courte »; étant courte, elle doit correspondre à la " description d'une action »; ou encore c'est une phrase qui est «sans indications, ni de précisions de lieux, temps... ». On retrouve cette dimension sémantique voire communicative dans de nombreuses justifications, ainsi «Elle ne présente pas de double sens ", "Une phrase simple c'est une phrase simple qui désigne une seule action », "C'est la seule phrase simple avec une seule information ", "Dans cette phrase il n'y a aucune information supplémentaire», "Cette phrase donne le moins de renseignements ». Cette focalisation sur la dimension sémantique et pragmatique se retrouve chez de nombreux étudiants qui jugent de la simplicité d'une phrase à l'accessibilité du sens, censément complexifié par le recours à des figures de styles par exemple: «C'est une phrase simple, réelle sans métaphore donc on peut facilement se l'imaginer".

43 Cette même idée de simplicité de la phrase (dans son sens ordinaire donc) conduit certains étudiants à préciser ce qu'il n'y a pas : «Il n'y a pas de COI », « La phrase n'a pas de virgule, qui permet de donner des précisions de lieu, de temps comme les compléments circonstanciels de lieu ou de temps ». La phrase simple devient donc celle dont on peut retirer le moins d'éléments : « Tous les mots sont essentiels à la sémantique de cette phrase ».

L'ensemble des concepts associés à l'idée que les étudiants se font de la simplicité en grammaire est susceptible d'être mobilisé. Ainsi, le critère morphologique est convoqué et la phrase est jugée simple, car «Le temps est celui du présent de l'indicatif», «Les temps sont au présent, phrase simple ».

45 L'introduction de modalisateurs tels que « moins » ou " plus » donne à voir comment ce qui est évalué est bien un degré de similitude avec le stéréotype de la complexité («moins complexe ») et de la simplicité (" plutôt simple »). Ces modalisateurs correspondent à des approximants (Pagnier, 2010) permettant de «jouer» avec les frontières des catégories ainsi sans cesse renégociées par ce jugement de typicalité.

À rebours, la catégorisation en conditions nécessaires et suffisantes conduit à distinguer nettement des «phrases simples » et des «phrases complexes » sur la base de propriétés jugées nécessaires et suffisantes.

47 Ce mécanisme de catégorisation, ou plus exactement de nomination (Pagnier, 2010) par approximation, par négociation des frontières catégorielles est très souvent mobilisé par les étudiants dans leur verbalisation des procédures. Ainsi, la phrase peut être " relativement ${ }^{9}$ simple », "C'est la phrase qui est la plus ${ }^{10}$ simple », "C'est la phrase qui me semble ${ }^{11}$ la plus simple». 

manière dont la nature des difficultés des futurs PE n'est immédiatement perceptible ni par les étudiants eux-mêmes ni pour les enseignants qui les forment. En effet, le malentendu quant aux finalités et plus largement à l'inscription de leur pratique dans le champ de la littéracie scolaire est difficilement perceptible tant que ne sont pas interrogées les procédures et les catégories mobilisées. En cas de bonne réponse à une question par exemple, ni l'étudiant ni le formateur n'accède au mode de raisonnement, aux procédures mises en place pour répondre (correctement) à la question et il se peut (et nous l'avons montré) que le cheminement intellectuel ne soit pas celui attendu malgré la bonne réponse fournie. De même les « réponses formelles » recueillies pourraient laisser croire que la finalité de l'activité est effectivement identifiée. La commune focalisation sur la dimension résultative et matérielle de l'activité grammaticale explique certainement comment, dans bien des cas, enseignants et étudiants ont au fil des scolarités co-construit les conditions de ce malentendu entretenu jusqu'en master. langue et au savoir permet d'agir sur les représentations et la construction du savoir, en l'occurrence grammatical. Ce déplacement qui engage les apprenants d'une littéracie 
" ordinaire» à une littéracie "scolaire» ne se laisse pas aisément mesurer. Une expérimentation plus longue qui en interroge les conditions d'efficience est donc prévue.

\section{BIBLIOGRAPHIE}

BARTON, David \& HAMILTON, Mary. (2010). La littératie : une pratique sociale. Langage et société, 133, $45-62$.

BAUTIER, Élisabeth. (2002). Du rapport au langage : question d'apprentissages différenciés ou de didactique? Pratiques, 113-114, 41-54.

BAUTIER, Élisabeth \& RAYOU, Patrick. (2009). Les inégalités d'apprentissage. Programmes, pratiques et malentendus scolaires. Paris : PUF.

BELIN, Emmanuel. (2002). Une sociologie des espaces potentiels. Logique dispositive et expérience ordinaire. Bruxelles : De Boeck Université.

DELARUE-BRETON, Catherine. (2012a). Discours scolaire et paradoxe. Louvain : Academia/

L'Harmattan.

DelARUE-BRETON, Catherine. (2012b). Dispositifs et logique dispositive : perception des enjeux et inégalités scolaires. Dans M.-L. Élalouf, A. Robert, A. Belhadjin \& M.-F. Bishop (dir.), Les didactiques en question : état des lieux et perspectives pour la recherche et la formation (p. 120-130). Bruxelles : De Boeck Université.

DELARUE-BRETON, Catherine. (2014). Dialogue scolaire, dialogisme et évènements d'énonciation : malentendus et in-entendus au sein de la classe. Études de linguistiques appliquées, 173, 87-97.

DelARUe-Breton, Catherine \& BAUTIER, Élisabeth. (2015). Nouvelles littératies scolaires et inégalité des élèves : une production de significations différenciées. Le français aujourd'hui, 190, 51-60.

Elalouf, Marie-Laure. (2005). De la $6^{\mathrm{e}}$ à la $1^{\mathrm{re}}$, comment mobilisent-ils leurs connaissances sur la langue dans des tâches d'explication ? Pratiques, 125-126, 157-178.

FRAENKEL, Béatrice \& MBODJ, Aïssatou. (2010). Introduction. Les New Literacy studies, jalons historiques et perspectives actuelles. Langage et société, 133, 7-24.

HUBERT, Bruno. (2016). Formation des enseignants : le casse-tête de la grammaire. Le français aujourd'hui, 192, 117-129.

Hymes, Dell. (1962). The Ethnography of Speaking. Dans T. Gladwin \& W. C. Sturtevant (dir.), Anthropology and Human Behavior (p. 13-53). Washington, DC : Anthropological Society of Washington.

HYMES, Dell. (1972). Models of the Interaction of Language and Social Life. Dans J. J. Gumperz \& D. Hymes (dir.), Directions in Sociolinguistics (p. 35-71). Oxford : Blackwell.

HYMES, Dell. (1984). Vers la compétence de communication. Paris : Hatier/Didier.

KLEIBER, Georges. (2001). Remarques sur la dénomination. Cahiers de praxématique, 36, 21-41. 
PAGNIER, Thierry. (2010). La nomination des couleurs, des bruits et des odeurs par des élèves de 7 à 9 ans. Une lecture sociolinguistique du concept de nomination. Synergies Roumanie, 5, 195-214.

Richard-Principalli, Patricia, Ferone, Georges \& Delarue-Breton, Catherine. (2016). L'écriture dans les forums de discussion : entre genre premier et genre second. Recherches textuelles, 13 , 399-416.

RocHEX, Jean-Yves \& CRINON, Jacques (dir.). (2011). La construction des inégalités scolaires. Au cour des pratiques et des dispositifs d'enseignement. Rennes : PUR.

Ulma, Dominique. (2016). Construction de savoirs grammaticaux et conceptualisation. Le français aujourd'hui, 192, 97-106.

\section{NOTES}

1. Compte-tenu du format de cet article, nous ne pouvons que reprendre dans le corps du texte certaines questions et réponses recueillies soit lors du test de grammaire soit pendant les entretiens. Par ailleurs, les résultats proposés ici ne sont pas systématiques dans la mesure où la recherche en est à un stade exploratoire. Elle se poursuit en 2017-2018, nos résultats seront donc complétés et systématisés dans les publications à venir.

2. Par exemple : «À votre avis, pourquoi on vous a fait faire ce type de test? Qu'est-ce qu'on voulait que vous appreniez avec ces questions? Qu'est-ce qu'on vise?»; mais également à la question «quelle est la fonction de l'élément en gras » dans la phrase «Aux yeux de tous, mon père reste un médecin dévoué » : qu'est-ce qu'on cherchait à vérifier ? ".

3. «Sélectionnez la ou les segmentations possibles pour cette phrase qui peut recevoir deux sens. »

4. Souligné par nous-mêmes.

5. Par exemple sous forme de questions du type «Qu'est-ce que vous faites quand vous dites que vous cherchez? Comment vous y prenez-vous? Qu'est-ce que vous recherchez ? Pourquoi ? Quel (s) indice(s) significatif(s) recherchez-vous/prenez-vous en compte?».

6. Par exemple «Qu'est-ce que vous faites quand vous dites que vous lisez? Qu'est-ce que c'est que lire? Que faites-vous concrètement quand vous lisez (annotation, soulignement/ surlignement)?».

7. Ou ne visant pas directement la construction de significations («avoir une bonne note», « réussir son concours », etc.).

8. Selon Kleiber (2001), la dénomination est caractérisée par l'« établissement d'une règle de fixation référentielle » dont ne bénéficie pas la désignation. La désignation est donc définie en creux comme tout lien référentiel ne bénéficiant pas de cette stabilité. Kleiber propose ainsi de différencier les énoncés dénominatifs qui acceptent les formules « $X$ est le nom de $\mathrm{X}$ » et « $\mathrm{X}$ s'appelle $X »$ ( $p$. ex. $X=$ moucheron et $\mathrm{X}=$ petite mouche) des énoncés de désignation qui, eux, n'autorisent pas les deux formules précédentes. Kleiber explique ce phénomène par le codage préalable qui caractérise le lien référentiel construit par la dénomination.

9. Souligné par nous-mêmes.

10. Idem.

11. Idem.

12. Propos extraits des entretiens réalisés en binôme ou trinôme. 


\section{RÉSUMÉS}

Nous proposons de transposer la notion de co-construction des inégalités et de malentendus sociocognitifs développés au sein du CIRCEFT au contexte de formation universitaire des futurs professeurs des écoles. Le concept de malentendu sociocognitif invite à prendre en compte le travail interprétatif de l'apprenant. C'est cette préoccupation qui nous a conduits à nous intéresser aux literacy studies et à chercher à mettre en évidence le sens que les étudiants ont effectivement construit des situations d'enseignement-apprentissage de la grammaire.

Ainsi, les étudiants ne semblent pas inscrire ces « évènements de littéracie » dans les pratiques spécifiques de littéracie que constitue justement ce que l'on nomme la littéracie scolaire. Au-delà du sens que les étudiants donnent aux catégories grammaticales (sujet, phrase, verbe, etc.), nous nous sommes intéressés au sens qu'ils donnent à des opérations linguistiques (segmentation, suppression, déplacement, commutation) et aux enjeux d'apprentissage de l'activité grammaticale en analysant comment ils utilisent ces manipulations linguistiques et ces catégories.

We propose to translate the concept of co-construction of inequalities and of sociocognitive misunderstanding developed within the CIRCEFT to the context of university training of future teachers of schools. The concept of sociocognitive misunderstanding invites to take into account the interpretative work of the learner. It is this concern that has led us to take an interest in Literacy Studies. From these concepts, we highlight the meaning that the students have actually constructed of situations of teaching-learning of grammar.

Thus, students do not seem to be putting these "literacy events" in the specific literacy practices of what is called school literacy. Beyond the meaning that students give to the grammatical categories (subject, sentence, verb, etc.), we are interested in the meaning they give to linguistic operations (segmentation, suppression, displacement, commutation) and learning issues of grammatical activity by analyzing how they use these linguistic manipulations and categories.

\section{INDEX}

Keywords : literacy event, scholar literacy, grammar, categorization, sociocognitive misunderstanding, coconstruction of inequality

Mots-clés : (évènement de) littéracie (scolaire), grammaire, malentendus sociocognitifs, coconstruction des inégalités, catégorisation

\section{AUTEURS}

\section{BELINDA LAVIEU-GWOZDZ}

Université Paris-Est Créteil, EA 4384 CIRCEFT-Escol*

(* Centre interuniversitaire de recherches sur la culture, l'éducation, la formation, le travail, équipe Éducation-scolarisation, universités Paris 8 et Paris-Est Créteil) 


\section{THIERRY PAGNIER}

Université Paris-Est Créteil, EA 4384 CIRCEFT-Escol*

(* Centre interuniversitaire de recherches sur la culture, l'éducation, la formation, le travail, équipe Éducation-scolarisation, universités Paris 8 et Paris-Est Créteil) 